\title{
PRECISE LARGE DEVIATIONS FOR SUMS OF RANDOM VARIABLES WITH CONSISTENTLY VARYING TAILS IN MULTI-RISK MODELS
}

\author{
SHIJIE WANG, ${ }^{* *}$ East China Normal University and Anhui University \\ WENSHENG WANG, ${ }^{* * *}$ East China Normal University
}

\begin{abstract}
Assume that there are $k$ types of insurance contracts in an insurance company. The $i$ th related claims are denoted by $\left\{X_{i j}, j \geq 1\right\}, i=1, \ldots, k$. In this paper we investigate large deviations for both partial sums $S\left(k ; n_{1}, \ldots, n_{k}\right)=\sum_{i=1}^{k} \sum_{j=1}^{n_{i}} X_{i j}$ and random sums $S(k ; t)=\sum_{i=1}^{k} \sum_{j=1}^{N_{i}(t)} X_{i j}$, where $N_{i}(t), i=1, \ldots, k$, are counting processes for the claim number. The obtained results extend some related classical results.

Keywords: Large deviation; loss process; consistently varying tail; sums of random variables
\end{abstract}

2000 Mathematics Subject Classification: Primary 60F10; 60F05; 60G50

\section{Introduction}

In a classical insurance risk model the surplus is described as the initial surplus plus the premium income with the claims taken off. Large deviations for the loss process of a classical insurance risk model have been widely investigated. Let $\left\{X_{j}, j \geq 1\right\}$ be a sequence of independent and identically distributed (i.i.d.) nonnegative random variables with common distribution function $F(x)=\mathrm{P}(X \leq x)$ which denote the claims (or the loss amounts), and let $N(t)$ denote a nonnegative integer-valued counting process for the claim number. Here, $N(t)$ can be a Poisson process, Cox process, or a marked point process. We assume that $N(t)$ is independent of $\left\{X_{j}, j \geq 1\right\}, 0<\mathrm{E} X_{1}=: \mu<\infty$ (i.e. it denotes the expected claim amounts, assumed to equal the premium), and that $\mathrm{E} N(t)=: \lambda(t) \rightarrow \infty$ as $t \rightarrow \infty$. If the premium $\mu$ is 'fair' and the interest rate is 0 then the loss process of the insurance company within the period $[0, t]$ is described as

$$
W(t)=\sum_{j=1}^{N(t)}\left(X_{j}-\mu\right) .
$$

If the company has the initial reserve $x$, the surplus process can be written as $U(t)=x-$ $W(t)$. Thus, the large deviation probabilities of the loss process can be used to characterize the ruin probability asymptotically, which is a very important objective in risk management. By convention, we write $S(t)=\sum_{j=1}^{N(t)} X_{j}$. Mainstream research on precise large deviation probabilities has concentrated on the study of the asymptotic

$$
\mathrm{P}(S(t)-\mu \lambda(t)>x) \sim \lambda(t) \bar{F}(x),
$$

\footnotetext{
Received 30 April 2007; revision received 28 August 2007.

* Postal address: Department of Statistics, East China Normal University, Shanghai 200062, P. R. China.

** Email address: ahuwsj@126.com

*** Email address: wswang@stat.ecnu.edu.cn
} 
which holds uniformly for some $x$-region $\mathscr{D}(t)$. Here and throughout the paper, we let $\bar{F}(x):=$ $1-F(x)$, and the uniformity is understood in the following sense:

$$
\lim _{t \rightarrow \infty} \sup _{x \in \mathcal{D}(t)}\left|\frac{\mathrm{P}(S(t)-\mu \lambda(t)>x)}{\lambda(t) \bar{F}(x)}-1\right|=0 .
$$

Some earlier work on large deviations can be found, for example, in Nagaev (1969) and Heyde (1967). Nagaev (1973), (1979) studied the large deviation probabilities (1.1) for the claims with regularly varying tails. Cline and Hsing (1991) and Klüppelberg and Mikosch (1997) extended the results to the so-called ERV (extended regularly varying) class. Recently $\mathrm{Ng}$ et al. (2004) studied the precise large deviation for sums of claims with consistently varying tails, which extended the asymptotic result to a larger subclass of heavy-tailed distributions.

However, in the literature to date all large deviation results for loss processes have been studied for only one kind of claim. That is to say they always assume that the company provides only one kind of insurance contract. In reality this assumption is not correct, so the large deviation problem of multi-risk models is more valuable. In this paper we assume that the company has $k$ types of insurance contracts. The $i$ th related loss amounts are denoted by $\left\{X_{i j}, j \geq 1\right\}$, which are i.i.d. nonnegative random variables with common distribution function $F_{i}(x)$ that has positive finite expectation $\mu_{i}, i=1, \ldots, k$. Let $\left\{N_{i}(t), i=1, \ldots, k\right\}$ denote a nonnegative integer-valued counting processes for the claim number. We assume that $\left\{X_{i j}, j \geq 1\right\}_{i=1}^{k}$ and $\left\{N_{i}(t), i=1, \ldots, k\right\}$ are mutually independent, and that $\mathrm{E} N_{i}(t)=$ $\lambda_{i}(t) \rightarrow \infty$ as $t \rightarrow \infty, i=1, \ldots, k$. Assume that $\bar{F}_{i}(x)>0$ for all $x \in(-\infty, \infty)$ and $i=1, \ldots, k$. Let

$$
S(k ; t)=\sum_{i=1}^{k} \sum_{j=1}^{N_{i}(t)} X_{i j}, \quad t \geq 0 .
$$

We can easily see that if all $F_{i}(x), i=1, \ldots, k$, are the same distribution function then $S(k ; t)$ is the one-risk model with $N_{1}(t)$ replacing $\sum_{i=1}^{k} N_{i}(t)$. In this sense $S(k ; t)$ is a natural generalization of $S(t)$. The aim of this paper is to investigate the precise large deviations for $S(k ; t)$. Up to now, to the best of our knowledge, little is known about the large deviations for such random sums with different kinds of distributions because the known methods to study large deviations for one-risk models do not work for $S(k ; t), k \geq 2$, any longer. Our results extend those of $\mathrm{Ng}$ et al. (2004) to multi-risk models.

The rest of this paper is organized as follows. In Section 2 we present some notation and preliminaries. In Section 3 we prove large deviations for nonrandom sums of random variables with consistently varying tails in multi-risk models. In Section 4 we investigate large deviations for random sums of random variables with consistently varying tails in multi-risk models. An application of our main results is stated in Section 5 .

\section{Notation and preliminaries}

In insurance and finance models the sums of heavy-tailed random variables are very important. In this section we assume that all distribution functions have finite mean. We say that a nonnegative random variable $X$ (or its distribution function $F$ ) is heavy tailed if it has no finite exponential moments. The most important heavy-tailed subclass is the subexponential class (denoted by $\&$ ). By definition, a distribution function $F$ supported on $[0, \infty)$ is in $\&$ if and only 
if

$$
\lim _{x \rightarrow \infty} \frac{\overline{F^{n *}}(x)}{\bar{F}(x)}=n \text { for all } n \geq 2
$$

(or equivalently for some $n \geq 2$ ), where $F^{n *}$ denotes the $n$-fold convolution of $F$. Another well-known heavy-tailed subclass is called the dominated variation class (denoted by $\mathscr{D})$ ). A distribution function $F$ supported on $(-\infty, \infty)$ is in $\mathscr{D}$ if and only if

$$
\limsup _{x \rightarrow \infty} \frac{\bar{F}(\theta x)}{\bar{F}(x)}<\infty \text { for any } 0<\theta<1
$$

(or equivalently for some $0<\theta<1$ ). A useful sharp inequality about tail probabilities of sums of random variables with dominated varying tails can be found in Tang and Yan (2002). A random variable is said to have a regularly varying tail if its distribution $F$ satisfies

$$
\lim _{x \rightarrow \infty} \frac{\bar{F}(x y)}{\bar{F}(x)}=y^{-\alpha}
$$

for any $y>0$ and some $\alpha>1$. We use $\mathcal{R}_{-\alpha}$ to denote this class of random variables. A distribution $F$ with support on $[0, \infty)$ is said to be in $\operatorname{ERV}(-\alpha,-\beta)$ for some $\alpha, \beta$, with $0<\alpha \leq \beta<\infty$, if

$$
y^{-\beta} \leq \liminf _{x \rightarrow \infty} \frac{\bar{F}(x y)}{\bar{F}(x)} \leq \limsup _{x \rightarrow \infty} \frac{\bar{F}(x y)}{\bar{F}(x)} \leq y^{-\alpha} \quad \text { for any } y>1 .
$$

For more details on regularly varying tails and extended regularly varying tails, see Klüppelberg and Mikosch (1997) or Tang et al. (2001). Recently, Ng et al. (2004) considered a subclass of heavy-tailed distributions slightly larger than the ERV class, called class $\mathcal{C}$. We restate their definition as follows.

Definition 2.1. A distribution function $F$ supported on $[0, \infty)$ belongs to $\mathcal{C}$ if

$$
\lim _{y \downarrow 1} \liminf _{x \rightarrow \infty} \frac{\bar{F}(x y)}{\bar{F}(x)}=1 \quad \text { or equivalently } \quad \lim _{y \uparrow 1} \limsup _{x \rightarrow \infty} \frac{\bar{F}(x y)}{\bar{F}(x)}=1 .
$$

Such a distribution function $F$ is usually said to have a consistently varying tail. The class $\mathcal{C}$ was also thoroughly studied by Berman (1982), who called it 'regular oscillation' and by Cline (1994), who called it 'intermediate regular variation'.

Obviously, if $F \in \mathscr{D}$ then, for any $y>0, \bar{F}(x y)$ and $\bar{F}(x)$ are of the same order as $x$ tends to $\infty$ in the sense that

$$
0<\liminf _{x \rightarrow \infty} \frac{\bar{F}(x y)}{\bar{F}(x)} \leq \limsup _{x \rightarrow \infty} \frac{\bar{F}(x y)}{\bar{F}(x)}<\infty .
$$

Set

$$
\gamma_{F}:=\inf \left\{-\frac{\log \gamma(y)}{\log y}, y>1\right\},
$$

where $\gamma(y)=\liminf _{x \rightarrow \infty}(\bar{F}(x y) / \bar{F}(x))$. In the terminology of Bingham et al. (1987), $\gamma_{F}$ is called the upper Matuszewska index of the distribution function $F$. If $F \in \operatorname{ERV}(-\alpha,-\beta)$ then $\alpha<\gamma_{F}<\beta$. It is easy to check that, whenever $0<\alpha \leq \gamma \leq \beta<\infty$, we have the following inclusion relationship:

$$
\mathcal{R}_{-\gamma} \subset \operatorname{ERV}(-\alpha,-\beta) \subset \mathcal{C} \subset \mathscr{D} \cap \S
$$




\section{Large deviations for nonrandom sums}

In this section we shall provide large deviations for nonrandom sums. Let $k$ be a positive integer, and let $\left\{n_{i}, i=1, \ldots, k\right\}$ be $k$ positive integer sequences. For simplicity, we use the notation

$$
S_{n_{i}}=\sum_{j=1}^{n_{i}} X_{i j}, \quad i=1, \ldots, k, \quad S\left(k ; n_{1}, \ldots, n_{k}\right)=\sum_{i=1}^{k} \sum_{j=1}^{n_{i}} X_{i j}
$$

therefore, $S\left(k ; n_{1}, \ldots, n_{k}\right)=\sum_{i=1}^{k} S_{n_{i}}$. We can easily see that

$$
\operatorname{ES}\left(k ; n_{1}, \ldots, n_{k}\right)=\sum_{i=1}^{k} n_{i} \mu_{i}
$$

Theorem 3.1. For $i=1, \ldots, k$, let $\left\{X_{i j}, j \geq 1\right\}$ be i.i.d. nonnegative random variables with common distribution function $F_{i}(x)$ and finite expectation $\mu_{i}>0$, and let $\left\{n_{i}\right\}$ be a positive integer sequence. We assume that $\left\{X_{i j}, j \geq 1\right\}_{i=1}^{k}$ are mutually independent. If $F_{i} \in \mathcal{C}$ for all $i=1, \ldots, k$ then, for any fixed $\gamma>0$, we find that $\left(\right.$ as $n_{i} \rightarrow \infty$ for all $i=1, \ldots, k$ )

$$
\mathrm{P}\left(S\left(k ; n_{1}, \ldots, n_{k}\right)-\sum_{i=1}^{k} n_{i} \mu_{i}>x\right) \sim \sum_{i=1}^{k} n_{i} \bar{F}_{i}(x)
$$

holds uniformly for all $x \geq \max \left\{\gamma n_{i}, i=1, \ldots, k\right\}:=\Delta(k)$, that is

$$
\lim _{n_{1}, \ldots, n_{k} \rightarrow \infty} \sup _{x \geq \Delta(k)}\left|\frac{P\left(S\left(k ; n_{1}, \ldots, n_{k}\right)-\sum_{i=1}^{k} n_{i} \mu_{i}>x\right)}{\sum_{i=1}^{k} n_{i} \bar{F}_{i}(x)}-1\right|=0 .
$$

Remark 3.1. If all $F_{i}(x), i=1, \ldots, k$, are the same distribution function then (3.1) implies Theorem 3.1 of $\mathrm{Ng}$ et al. (2004).

Remark 3.2. By Theorem 2.2 of Cai and Tang (2004), if $F_{i} \in \mathcal{C}$ then $\overline{F_{i}^{n_{i} *}} \in \mathcal{C}$. Thus, (3.1) holds in the nonuniform sense (with $n_{1}, \ldots, n_{k}$ fixed and $x \rightarrow \infty$ ). However, (3.1) in the uniform sense (see Theorem 3.1) cannot be derived directly.

Proposition 3.1, below, is Theorem 3.1 of $\mathrm{Ng}$ et al. (2004), which will be used to prove Theorem 3.1.

Proposition 3.1. ( $\mathrm{Ng}$ et al. (2004, Theorem 3.1).) Let $\left\{X_{j}, j \geq 1\right\}$ be a sequence of i.i.d. nonnegative random variables with common distribution function $F(x)$, which has finite expectation $\mu>0$. If $F \in \mathcal{C}$ then, for any fixed $\gamma>0$, as $n \rightarrow \infty$,

$$
\mathrm{P}\left(S_{n}-n \mu>x\right) \sim n \bar{F}(x) \text { uniformly for } x \in[\gamma n, \infty) .
$$

Proof of Theorem 3.1. We use induction to prove (3.1). For the case in which $k=2$, we first show that

$$
\liminf _{n_{1}, n_{2} \rightarrow \infty} \inf _{x \geq \Delta(2)} \frac{\mathrm{P}\left(S\left(2 ; n_{1}, n_{2}\right)-\sum_{i=1}^{2} n_{i} \mu_{i}>x\right)}{n_{1} \bar{F}_{1}(x)+n_{2} \bar{F}_{2}(x)} \geq 1
$$


Note that, for any $0<\varepsilon<1$ and any $x>0$,

$$
\begin{aligned}
& \mathrm{P}\left(S\left(2 ; n_{1}, n_{2}\right)-n_{1} \mu_{1}-n_{2} \mu_{2}>x\right) \\
&= \mathrm{P}\left(S_{n_{1}}+S_{n_{2}}>x+n_{1} \mu_{1}+n_{2} \mu_{2}\right) \\
& \geq \mathrm{P}\left(\left\{S_{n_{1}}>x+n_{1} \mu_{1}+\varepsilon n_{2} \mu_{2}, S_{n_{2}}>(1-\varepsilon) n_{2} \mu_{2}\right\}\right. \\
&\left.\quad \cup\left\{S_{n_{2}}>x+n_{2} \mu_{2}+\varepsilon n_{1} \mu_{1}, S_{n_{1}}>(1-\varepsilon) n_{1} \mu_{1}\right\}\right) \\
& \geq \mathrm{P}\left(S_{n_{1}}>x+n_{1} \mu_{1}+\varepsilon n_{2} \mu_{2}\right) \mathrm{P}\left(S_{n_{2}}>(1-\varepsilon) n_{2} \mu_{2}\right) \\
&+\mathrm{P}\left(S_{n_{2}}>x+n_{2} \mu_{2}+\varepsilon n_{1} \mu_{1}\right) \mathrm{P}\left(S_{n_{1}}>(1-\varepsilon) n_{1} \mu_{1}\right) \\
&-\mathrm{P}\left(S_{n_{1}}>x+n_{1} \mu_{1}+\varepsilon n_{2} \mu_{2}\right) \mathrm{P}\left(S_{n_{2}}>x+n_{2} \mu_{2}+\varepsilon n_{1} \mu_{1}\right) .
\end{aligned}
$$

By Proposition 3.1, for any $0<\delta<1$, there exists a positive constant $m_{0}=m_{0}(\delta)$ such that, for any $n_{1} \geq m_{0}$,

$$
\sup _{x \geq \gamma n_{1}}\left|\frac{\mathrm{P}\left(S_{n_{1}}-n_{1} \mu_{1}>x+\varepsilon n_{2} \mu_{2}\right)}{n_{1} \bar{F}_{1}\left(x+\varepsilon n_{2} \mu_{2}\right)}-1\right|<\delta .
$$

Conversely, the classical law of large numbers implies that, for any $0<\varepsilon<1$,

$$
\mathrm{P}\left(S_{n_{1}}>(1-\varepsilon) n_{1} \mu_{1}\right) \rightarrow 1 .
$$

Thus, for any $0<\delta<1$, there exists a positive constant $m_{1}=m_{1}(\delta)$ such that, for any $n_{1} \geq m_{1}$,

$$
\mathrm{P}\left(S_{n_{1}}>(1-\varepsilon) n_{1} \mu_{1}\right)>1-\delta .
$$

Similarly, it is easy to see that analogous results to (3.5) and (3.6) hold for $S_{n_{2}}$. Combining these arguments, we find that, for any $0<\delta<1$, there exist large enough positive constants $m^{*}$ such that, for any $n_{1} \geq m^{*}$, any $n_{2} \geq m^{*}$, and any $x \geq \Delta(2)$,

$$
\begin{aligned}
& \mathrm{P}\left(S\left(2 ; n_{1}, n_{2}\right)-n_{1} \mu_{1}-n_{2} \mu_{2}>x\right) \\
& \geq(1-\delta)^{2} n_{1} \bar{F}_{1}\left(x+\varepsilon n_{2} \mu_{2}\right)+(1-\delta)^{2} n_{2} \bar{F}_{2}\left(x+\varepsilon n_{1} \mu_{1}\right) \\
& \quad-(1+\delta)^{2} n_{1} \bar{F}_{1}\left(x+\varepsilon n_{2} \mu_{2}\right) n_{2} \bar{F}_{2}\left(x+\varepsilon n_{1} \mu_{1}\right) \\
& \geq(1-\delta)^{2} n_{1} \bar{F}_{1}\left(x+\varepsilon n_{2} \mu_{2}\right)+(1-\delta)^{2} n_{2} \bar{F}_{2}\left(x+\varepsilon n_{1} \mu_{1}\right)-o\left(n_{1} \bar{F}_{1}(x)+n_{2} \bar{F}_{2}(x)\right)
\end{aligned}
$$

uniformly for $x \geq \Delta(2)$, where to obtain the last inequality we used the following facts (by Proposition 3.1): $n_{1} \bar{F}_{1}(x)=o(1)$ and $n_{2} \bar{F}_{2}(x)=o(1)$ uniformly for $x \geq \Delta(2)$, as $n_{1}$ and $n_{2}$ tend to $\infty$.

Now we show that

$$
\lim _{\varepsilon \downarrow 0} \lim _{n_{2} \rightarrow \infty} \sup _{x \geq \gamma n_{2}}\left|\frac{\bar{F}_{1}\left(x+\varepsilon n_{2} \mu_{2}\right)}{\bar{F}_{1}(x)}-1\right|=0 .
$$

Clearly, since $\overline{F_{1}}$ is nonincreasing, we have, for all $x \geq \gamma n_{2}$,

$$
\bar{F}_{1}\left(\left(1+\frac{\varepsilon \mu_{2}}{\gamma}\right) x\right) \leq \bar{F}_{1}\left(x+\varepsilon n_{2} \mu_{2}\right) \leq \bar{F}_{1}(x) .
$$

We can easily see that

$$
\lim _{\varepsilon \downarrow 0} \limsup _{n_{2} \rightarrow \infty} \sup _{x \geq \gamma n_{2}} \frac{\bar{F}_{1}\left(x+\varepsilon n_{2} \mu_{2}\right)}{\bar{F}_{1}(x)} \leq 1 .
$$


Note that

$$
\inf _{x \geq \gamma n_{2}} \frac{\bar{F}_{1}\left(x+\varepsilon n_{2} \mu_{2}\right)}{\bar{F}_{1}(x)} \geq \inf _{x \geq \gamma n_{2}} \frac{\bar{F}_{1}\left(\left(1+\varepsilon \mu_{2} / \gamma\right) x\right)}{\bar{F}(x)} .
$$

The definition of $\mathcal{C},(2.1)$, yields

$$
\lim _{\varepsilon \downarrow 0} \liminf _{n_{2} \rightarrow \infty} \inf _{x \geq \gamma n_{2}} \frac{\bar{F}_{1}\left(x+\varepsilon n_{2} \mu_{2}\right)}{\bar{F}_{1}(x)} \geq \lim _{\varepsilon \downarrow 0} \liminf _{n_{2} \rightarrow \infty} \inf _{x \geq \gamma n_{2}} \frac{\bar{F}_{1}\left(\left(1+\varepsilon \mu_{2} / \gamma\right) x\right)}{\bar{F}_{1}(x)} \geq 1 .
$$

By (3.9) and (3.10), we obtain (3.8).

By (3.8) we find that, for any $0<\delta<1$ such that for all sufficiently small $\varepsilon$, all sufficiently large $n_{2}$, and all $x \geq \gamma n_{2}$,

$$
\bar{F}_{1}\left(x+\varepsilon n_{2} \mu_{2}\right) \geq(1-\delta) \bar{F}_{1}(x) .
$$

Similarly, we find that, for any $0<\delta<1$ such that for all sufficiently small $\varepsilon$, all sufficiently large $n_{1}$, and all $x \geq \gamma n_{1}$,

$$
\bar{F}_{2}\left(x+\varepsilon n_{1} \mu_{1}\right) \geq(1-\delta) \bar{F}_{2}(x) .
$$

By (3.7), (3.11), and (3.12), we arrive at

$$
\begin{aligned}
& \mathrm{P}\left(S\left(2 ; n_{1}, n_{2}\right)-n_{1} \mu_{1}-n_{2} \mu_{2}>x\right) \\
& \quad \geq(1-\delta)^{3}\left(n_{1} \bar{F}_{1}(x)+n_{2} \bar{F}_{2}(x)\right)-o\left(n_{1} \bar{F}_{1}(x)+n_{2} \bar{F}_{2}(x)\right) .
\end{aligned}
$$

Therefore, letting $\delta \downarrow 0$, we obtain (3.3).

Next we show that

$$
\limsup _{n_{1}, n_{2} \rightarrow \infty} \sup _{x \geq \Delta(2)} \frac{\mathrm{P}\left(S\left(2 ; n_{1}, n_{2}\right)-n_{1} \mu_{1}-n_{2} \mu_{2}>x\right)}{n_{1} \bar{F}_{1}(x)+n_{2} \bar{F}_{2}(x)} \leq 1 .
$$

Note that, for any $0<\varepsilon<\frac{1}{2}$ and any $x>0$, by Proposition 3.1, we have

$$
\begin{aligned}
\mathrm{P}(S(2) & \left.\left.n_{1}, n_{2}\right)-n_{1} \mu_{1}-n_{2} \mu_{2}>x\right) \\
= & \mathrm{P}\left(S_{n_{1}}+S_{n_{2}}>x+n_{1} \mu_{1}+n_{2} \mu_{2}\right) \\
\leq & \mathrm{P}\left(\left\{S_{n_{1}}>(1-\varepsilon) x+n_{1} \mu_{1}\right\} \cup\left\{S_{n_{2}}>(1-\varepsilon) x+n_{2} \mu_{2}\right\}\right. \\
& \left.\quad \cup\left\{S_{n_{1}}>\varepsilon x+n_{1} \mu_{1}, S_{n_{2}}>\varepsilon x+n_{2} \mu_{2}\right\}\right) \\
\leq & \mathrm{P}\left(S_{n_{1}}>(1-\varepsilon) x+n_{1} \mu_{1}\right)+\mathrm{P}\left(S_{n_{2}}>(1-\varepsilon) x+n_{2} \mu_{2}\right) \\
& +\mathrm{P}\left(S_{n_{1}}>\varepsilon x+n_{1} \mu_{1}\right) \mathrm{P}\left(S_{n_{2}}>\varepsilon x+n_{2} \mu_{2}\right) \\
\leq & (1+\delta) n_{1} \bar{F}_{1}((1-\varepsilon) x)+(1+\delta) n_{2} \bar{F}_{2}((1-\varepsilon) x) \\
& +(1+\delta)^{2} n_{1} \bar{F}_{1}(\varepsilon x) n_{2} \bar{F}_{2}(\varepsilon x) .
\end{aligned}
$$

In a similar way to the argument we used to obtain (3.8), we have

$$
\lim _{\varepsilon \downarrow 0} \lim _{n_{2} \rightarrow \infty} \sup _{x \geq \gamma n_{2}}\left|\frac{\bar{F}_{1}((1-\varepsilon) x)}{\bar{F}_{1}(x)}-1\right|=0
$$

and

$$
\lim _{\varepsilon \downarrow 0} \lim _{n_{1} \rightarrow \infty} \sup _{x \geq \gamma n_{1}}\left|\frac{\bar{F}_{2}((1-\varepsilon) x)}{\bar{F}_{2}(x)}-1\right|=0 .
$$


Conversely, note that

$$
\frac{n_{1} \bar{F}_{1}(\varepsilon x) n_{2} \bar{F}_{2}(\varepsilon x)}{n_{1} \bar{F}_{1}(x)+n_{2} \bar{F}_{2}(x)}=1 /\left(\frac{\bar{F}_{1}(x)}{\bar{F}_{1}(\varepsilon x)} \frac{1}{n_{2} \bar{F}_{2}(\varepsilon x)}+\frac{\bar{F}_{2}(x)}{\bar{F}_{2}(\varepsilon x)} \frac{1}{n_{1} \bar{F}_{1}(\varepsilon x)}\right) .
$$

By (3.2), it is easy to check that

$$
\lim _{n_{1} \rightarrow \infty} \sup _{x \geq \gamma n_{1}} n_{1} \bar{F}_{1}(\varepsilon x)=0 \text { and } \lim _{n_{2} \rightarrow \infty} \sup _{x \geq \gamma n_{2}} n_{2} \bar{F}_{2}(\varepsilon x)=0 .
$$

Moreover, since, by (2.4), $F_{1}, F_{2} \in \mathcal{C} \subset \mathcal{D}$, we have, by (2.2), for any $0<\varepsilon<1$,

$$
\liminf _{n_{1} \rightarrow \infty} \inf _{x \geq \gamma n_{1}} \frac{\bar{F}_{1}(x)}{\bar{F}_{1}(\varepsilon x)}>0 \text { and } \liminf _{n_{2} \rightarrow \infty} \inf _{x \geq \gamma n_{2}} \frac{\bar{F}_{2}(x)}{\bar{F}_{2}(\varepsilon x)}>0 .
$$

Therefore, we have, as $n_{1}, n_{2} \rightarrow \infty$,

$$
n_{1} \bar{F}_{1}(\varepsilon x) n_{2} \bar{F}_{2}(\varepsilon x)=o\left(n_{1} \bar{F}_{1}(x)+n_{2} \bar{F}_{2}(x)\right) \quad \text { uniformly for } x \geq \Delta(2) .
$$

Using a similar argument to that used to prove (3.3), by (3.14)-(3.17) we arrive at

$$
\begin{aligned}
& \mathrm{P}\left(S\left(2 ; n_{1}, n_{2}\right)-n_{1} \mu_{1}-n_{2} \mu_{2}\right) \\
& \quad \leq(1+\delta)^{2}\left(n_{1} \bar{F}_{1}(x)+n_{2} \bar{F}_{2}(x)\right)+o\left(n_{1} \bar{F}_{1}(x)+n_{2} \bar{F}_{2}(x)\right) .
\end{aligned}
$$

Therefore, letting $\delta \downarrow 0$, we obtain (3.13). Thus, (3.1) holds for $k=2$. Now suppose that (3.1) holds for $k-1$ and, as for $k$, using a similar argument to that in (3.4), Proposition 3.1, and the induction hypothesis, we find that, as $n_{i} \rightarrow \infty$ for all $i=1, \ldots, k$,

$$
\begin{aligned}
\mathrm{P}(S(k & \left.\left.; n_{1}, \ldots, n_{k}\right)-\sum_{i=1}^{k} n_{i} \mu_{i}>x\right) \\
= & \mathrm{P}\left(S_{n_{1}}+\cdots+S_{n_{k}}>x+\sum_{i=1}^{k} n_{i} \mu_{i}\right) \\
\geq & \mathrm{P}\left(S_{n_{1}}+\cdots+S_{n_{k-1}}>x+\sum_{i=1}^{k-1} n_{i} \mu_{i}+\varepsilon n_{k} \mu_{k}\right) \mathrm{P}\left(S_{n_{k}}>(1-\varepsilon) n_{k} \mu_{k}\right) \\
& +\mathrm{P}\left(S_{n_{k}}>x+n_{k} \mu_{k}+\varepsilon \sum_{i=1}^{k-1} n_{i} \mu_{i}\right) \mathrm{P}\left(S_{n_{1}}+\cdots+S_{n_{k-1}}>(1-\varepsilon) \sum_{i=1}^{k-1} n_{i} \mu_{i}\right) \\
& -\mathrm{P}\left(S_{n_{1}}+\cdots+S_{n_{k-1}}>x+\sum_{i=1}^{k-1} n_{i} \mu_{i}+\varepsilon n_{k} \mu_{k}\right) \\
& \quad \times \mathrm{P}\left(S_{n_{k}}>x+n_{k} \mu_{k}+\varepsilon \sum_{i=1}^{k-1} n_{i} \mu_{i}\right) \\
\sim & \sum_{i=1}^{k-1} n_{i} \bar{F}_{i}\left(x+\varepsilon n_{k} \mu_{k}\right)+n_{k} \bar{F}_{k}\left(x+\varepsilon \sum_{i=1}^{k-1} n_{i} \mu_{i}\right)+\sum_{i=1}^{k-1} n_{i} \bar{F}_{i}(x) n_{k} \bar{F}_{k}(x) \\
\sim & \sum_{i=1}^{k} n_{i} \bar{F}_{i}(x)+o\left(\sum_{i=1}^{k} n_{i} \bar{F}_{i}(x)\right)
\end{aligned}
$$


uniformly for $x \geq \Delta(k)$. In the last step we used the same argument as in (3.8). In (3.18) it is necessary to mention that

$$
\mathrm{P}\left(S_{n_{1}}+\cdots+S_{n_{k-1}}>(1-\varepsilon) \sum_{i=1}^{k-1} n_{i} \mu_{i}\right) \rightarrow 1 .
$$

In fact, note that $\mathrm{P}\left(S_{n_{i}}>(1-\varepsilon) n_{i} \mu_{i}\right) \rightarrow 1, i=1, \ldots, k-1$, by the law of large numbers; thus, (3.19) holds. Now, (3.18) implies that

$$
\liminf _{n_{1}, \ldots, n_{k} \rightarrow \infty} \inf _{x \geq \Delta(k)} \frac{\mathrm{P}\left(S\left(k ; n_{1}, \ldots, n_{k}\right)-\sum_{i=1}^{k} n_{i} \mu_{i}>x\right)}{\sum_{i=1}^{k} n_{i} \bar{F}_{i}(x)} \geq 1 .
$$

For the reverse inequality, using a similar argument to that used in (3.14), (3.15), Proposition 3.1, and the induction hypothesis, we easily obtain

$$
\limsup _{n_{1}, \ldots, n_{k} \rightarrow \infty} \sup _{x \geq \Delta(k)} \frac{\mathrm{P}\left(S\left(k ; n_{1}, \ldots, n_{k}\right)-\sum_{i=1}^{k} n_{i} \mu_{i}>x\right)}{\sum_{i=1}^{k} n_{i} \bar{F}_{i}(x)} \leq 1 .
$$

Combining (3.20) and (3.21), we obtain the desired result, and the proof of Theorem 3.1 is now complete.

\section{Large deviations for random sums}

In this section we study large deviations for random sums. We will use the notation of Section 1. Moreover, throughout this section, we let

$$
S_{N_{i}(t)}=\sum_{j=1}^{N_{i}(t)} X_{i j}, \quad i=1, \ldots, k
$$

and $S(k ; t)=\sum_{i=1}^{k} S_{N_{i}(t)}$. To state our results, we will need the following assumption, which was used by $\mathrm{Ng}$ et al. (2004), and is satisfied for many common counting processes, for example, the renewal counting process and the Cox process.

Assumption 4.1. For all $i=1, \ldots k$,

$$
\mathrm{E}_{i}^{\theta_{i}}(t) \mathbf{1}_{\left(N_{i}(t)>(1+\delta) \lambda_{i}(t)\right)}=O\left(\lambda_{i}(t)\right)
$$

holds for any $\delta>0$ and some $\theta_{i}>\gamma_{F_{i}}$, where $\gamma_{F_{i}}$ is defined as in (2.3).

Remark 4.1. By Lemma 2.4 of $\mathrm{Ng}$ et al. (2004) we can easily see that Assumption 4.1 implies that $N_{i}(t) / \lambda_{i}(t) \stackrel{\mathrm{P}}{\rightarrow} 1, i=1, \ldots, k$, where $\stackrel{\text { P }}{\rightarrow}$ ' denotes convergence in probability. See also Lemma 3.1 of $\mathrm{Ng}$ et al. (2003).

Theorem 4.1. For $i=1, \ldots, k$, let $\left\{X_{i j}, j \geq 1\right\}$ be i.i.d. nonnegative random variables with common distribution function $F_{i}(x)$ that has finite expectation $\mu_{i}>0$, and let $\left\{N_{i}(t)\right\}$ be a nonnegative integer-valued process. Assume that $\left\{X_{i j}, j \geq 1\right\}_{i=1}^{k}$ and $\left\{N_{i}(t), i=1, \ldots, k\right\}$ are mutually independent, $F_{i} \in \mathcal{C}$, and that $\left\{N_{i}(t), i=1, \ldots, k\right\}$ satisfies Assumption 4.1. Then, for any fixed $\gamma>0$, as $t \rightarrow \infty$,

$$
\mathrm{P}\left(S(k ; t)-\sum_{i=1}^{k} \mu_{i} \lambda_{i}(t)>x\right) \sim \sum_{i=1}^{k} \lambda_{i}(t) \bar{F}_{i}(x)
$$


uniformly for $x \geq \max \left\{\gamma \lambda_{i}(t), i=1, \ldots, k\right\}:=\Gamma(k)$, that is

$$
\lim _{t \rightarrow \infty} \sup _{x \geq \Gamma(k)}\left|\frac{\mathrm{P}\left(S(k ; t)-\sum_{i=1}^{k} \mu_{i} \lambda_{i}(t)>x\right)}{\sum_{i=1}^{k} \lambda_{i}(t) \bar{F}_{i}(x)}-1\right|=0 .
$$

Remark 4.2. If $F_{1}(x)=\cdots=F_{k}(x)$, our Theorem 4.1 implies Theorem 4.1 of $\mathrm{Ng}$ et al. (2004).

Proof of Theorem 4.1. Again by induction, as in the proof of Theorem 3.1, it is sufficient to show that (4.1) holds for $k=2$. We first show that

$$
\liminf _{t \rightarrow \infty} \inf _{x \geq \Gamma(2)} \frac{\mathrm{P}\left(S(2 ; t)-\lambda_{1}(t) \mu_{1}-\lambda_{2}(t) \mu_{2}>x\right)}{\lambda_{1}(t) \bar{F}_{1}(x)+\lambda_{2}(t) \bar{F}_{2}(x)} \geq 1 .
$$

Note that, for any $0<\delta<\min \left\{\gamma / \mu_{1}, \gamma / \mu_{2}, 1\right\}$ and any $x>0$,

$$
\begin{aligned}
& \mathrm{P}\left(S(2 ; t)-\lambda_{1}(t) \mu_{1}-\lambda_{2}(t) \mu_{2}>x\right) \\
&=\mathrm{P}\left(S_{N_{1}(t)}+S_{N_{2}(t)}>x+\lambda_{1}(t) \mu_{1}+\lambda_{2}(t) \mu_{2}\right) \\
& \geq \mathrm{P}\left(\left\{S_{N_{1}(t)}>x+\lambda_{1}(t) \mu_{1}+\delta \lambda_{2}(t) \mu_{2}, S_{N_{2}(t)}>(1-\delta) \lambda_{2}(t) \mu_{2}\right\}\right. \\
&\left.\quad \cup\left\{S_{N_{2}(t)}>x+\lambda_{2}(t) \mu_{2}+\delta \lambda_{1}(t) \mu_{1}, S_{N_{1}(t)}>(1-\delta) \lambda_{1}(t) \mu_{1}\right\}\right) \\
& \geq \mathrm{P}\left(S_{N_{1}(t)}>x+\lambda_{1}(t) \mu_{1}+\delta \lambda_{2}(t) \mu_{2}\right) \mathrm{P}\left(S_{N_{2}(t)}>(1-\delta) \lambda_{2}(t) \mu_{2}\right) \\
&+\mathrm{P}\left(S_{N_{2}(t)}>x+\lambda_{2}(t) \mu_{2}+\delta \lambda_{1}(t) \mu_{1}\right) \mathrm{P}\left(S_{N_{1}(t)}>(1-\delta) \lambda_{1}(t) \mu_{1}\right) \\
&-\mathrm{P}\left(S_{N_{1}(t)}>x+\lambda_{1}(t) \mu_{1}+\delta \lambda_{2}(t) \mu_{2}, S_{N_{2}(t)}>x+\lambda_{2}(t) \mu_{2}+\delta \lambda_{1}(t) \mu_{1}\right) .
\end{aligned}
$$

Applying the same approach used in the proof of Theorem 4.1 of $\mathrm{Ng}$ et al. (2004) with $\bar{F}(x)$ replaced by $\bar{F}_{1}\left(x+\delta \lambda_{2}(t) \mu_{2}\right)$, we easily conclude that

$$
\lim _{\delta \downarrow 0} \lim _{t \rightarrow \infty} \sup _{x \geq \gamma \lambda_{1}(t)}\left|\frac{\mathrm{P}\left(S_{N_{1}(t)}-\lambda_{1}(t) \mu_{1}>x+\delta \lambda_{2}(t) \mu_{2}\right)}{\lambda_{1}(t) \bar{F}_{1}(x)}-1\right|=0 .
$$

By the stochastic law of large numbers and Remark 4.1, we easily obtain

$$
\frac{1}{\lambda_{1}(t)} \sum_{j=1}^{N_{1}(t)} X_{1 j}=\frac{N_{1}(t)}{\lambda_{1}(t)} \frac{1}{N_{1}(t)} \sum_{j=1}^{N_{1}(t)} X_{1 j} \stackrel{\mathrm{P}}{\rightarrow} 1 .
$$

Thus,

$$
\mathrm{P}\left(S_{N_{1}(t)}>(1-\delta) \lambda_{1} \mu_{1}\right) \rightarrow 1 .
$$

We can estimate $S_{N_{2}(t)}$ similarly. Therefore, by (4.2) and (4.3), we easily find that, for any $\varepsilon>0$, any sufficiently large $t$, any sufficiently small $\delta$, and $x \geq \Gamma(2)$,

$$
\begin{aligned}
& \mathrm{P}\left(S(2 ; t)-\lambda_{1}(t) \mu_{1}-\lambda_{2}(t) \mu_{2}>x\right) \\
& \quad \geq(1-\varepsilon)^{2} \lambda_{1}(t) \bar{F}_{1}(x)+(1-\varepsilon)^{2} \lambda_{2}(t) \bar{F}_{2}(x)-(1+\varepsilon)^{2} \lambda_{1}(t) \bar{F}_{1}(x) \lambda_{2}(t) \bar{F}_{2}(x) \\
& \quad=(1-\varepsilon)^{2}\left(\lambda_{1}(t) \bar{F}_{1}(x)+\lambda_{2}(t) \bar{F}_{2}(x)\right)-o\left(\lambda_{1}(t) \bar{F}_{1}(x)+\lambda_{2}(t) \bar{F}_{2}(x)\right) .
\end{aligned}
$$

Letting $\varepsilon \downarrow 0$, we have

$$
\liminf _{t \rightarrow \infty} \inf _{x \geq \Gamma(2)} \frac{\mathrm{P}\left(S(2 ; t)-\lambda_{1}(t) \mu_{1}-\lambda_{2}(t) \mu_{2}>x\right)}{\lambda_{1}(t) \bar{F}_{1}(x)+\lambda_{2}(t) \bar{F}_{2}(x)} \geq 1 .
$$


We now show that

$$
\limsup _{t \rightarrow \infty} \sup _{x \geq \Gamma(2)} \frac{\mathrm{P}\left(S(2 ; t)-\lambda_{1}(t) \mu_{1}-\lambda_{2}(t) \mu_{2}>x\right)}{\lambda_{1}(t) \bar{F}_{1}(x)+\lambda_{2}(t) \bar{F}_{2}(x)} \leq 1 .
$$

Note that, for any $0<\varepsilon<\frac{1}{2}$ and any $x>0$, by the same argument as in (3.15) and (3.16), we have

$$
\begin{aligned}
\mathrm{P}\left(S(2 ; t)-\lambda_{1}(t) \mu_{1}-\lambda_{2}(t) \mu_{2}>x\right) \\
=\mathrm{P}\left(S_{N_{1}(t)}+S_{N_{2}(t)}>x+\lambda_{1}(t) \mu_{1}+\lambda_{2}(t) \mu_{2}\right) \\
\leq \mathrm{P}\left(\left\{S_{N_{1}(t)}>(1-\varepsilon) x+\lambda_{1}(t) \mu_{1}\right\} \cup\left\{S_{N_{2}(t)}>(1-\varepsilon) x+\lambda_{2}(t) \mu_{2}\right\}\right. \\
\quad \quad\left\{\left\{S_{N_{1}(t)}>\varepsilon x+\lambda_{1}(t) \mu_{1}, S_{N_{2}(t)}>\varepsilon x+\lambda_{2}(t) \mu_{2}\right\}\right) \\
\leq \mathrm{P}\left(S_{N_{1}(t)}>(1-\varepsilon) x+\lambda_{1}(t) \mu_{1}\right)+\mathrm{P}\left(S_{N_{2}(t)}>(1-\varepsilon) x+\lambda_{2}(t) \mu_{2}\right) \\
\quad+\mathrm{P}\left(S_{N_{1}(t)}>\varepsilon x+\lambda_{1}(t) \mu_{1}\right) \mathrm{P}\left(S_{N_{2}(t)}>\varepsilon x+\lambda_{2}(t) \mu_{2}\right) \\
\sim \lambda_{1}(t) \bar{F}_{1}((1-\varepsilon) x)+\lambda_{2}(t) \bar{F}_{2}((1-\varepsilon) x)+\lambda_{1}(t) \bar{F}_{1}(\varepsilon x) \lambda_{2}(t) \bar{F}_{2}(\varepsilon x) \\
\sim \lambda_{1}(t) \bar{F}_{1}(x)+\lambda_{2}(t) \bar{F}_{2}(x)+o\left(\lambda_{1}(t) \bar{F}_{1}(x)+\lambda_{2}(t) \bar{F}_{2}(x)\right) .
\end{aligned}
$$

Thus, we obtain (4.5).

Combining (4.4) and (4.5), (4.1) holds for $k=2$. The proof of Theorem 4.1 is complete.

\section{Applications}

In this section we give an example of an application of our main result. Assume that there are two types of insurance contracts in an insurance company. The first kind of related loss amounts, $X=\left\{X_{j}, j \geq 1\right\}$, are i.i.d. nonnegative random variables with common distribution $F \in \mathcal{C}$ and finite expectation $\mu$. Their occurrence times $\left\{\sigma_{j}, j \geq 1\right\}$ constitute an ordinary renewal counting process

$$
N_{1}(t)=\sup \left\{n \geq 1, \sigma_{n} \leq t\right\}, \quad t \geq 0,
$$

with $\lambda_{1}(t)=\mathrm{E} N_{1}(t)<\infty$ for any $t \geq 0$. Also, $\left\{I_{j}, j \geq 1\right\}$ is a sequence of Bernoulli random variables, and $I_{j}$ has a common expectation $q$, where $0<q \leq 1$, and $q$ is the claimoccurrence probability of the $j$ th policy, $j \geq 1$. Assume that the second kind of loss amounts, $\left\{Y_{j}, j \geq 1\right\}$, are also i.i.d. nonnegative random variables with distribution $G(\neq F) \in \mathcal{C}$ and finite expectation $v$. Let $N_{2}(t)=N(\Lambda(t))$ be a Cox process, where $N(t)$ is an ordinary renewal process which is generated by i.i.d. nonnegative random variables $\left\{Z_{j}, j \geq 1\right\}$ with $\mathrm{E} Z_{1}=1$, and let $\Lambda(t), t \geq 0$, be another right-continuous nondecreasing process with $\Lambda(0)=0$, independent of $N_{2}(t)$, and $\mathrm{P}(\Lambda(t)<\infty)=1$ for any $t \geq 0$. Suppose that the sequences $\left\{X_{j}, j \geq 1\right\},\left\{I_{j}, j \geq 1\right\},\left\{Y_{j}, j \geq 1\right\}$, and $\left\{N_{1}(t), t \geq 0\right\},\left\{N_{2}(t), t \geq 0\right\}$ are mutually independent and that the sequence $\left\{I_{j}, j \geq 1\right\}$ is negatively associated. Then the total claim amount up to time $t$ is

$$
S(t)=\sum_{j=1}^{N_{1}(t)} X_{j} I_{j}+\sum_{j=1}^{N_{2}(t)} Y_{j}, \quad t \geq 0 .
$$

In this section we assume that the insurance company has two different kinds of risk at one time. Therefore, (5.1) is an extension of the one-risk model which has been investigated in the literature; see, for example, Denuit et al. (2002) or Ng et al. (2004). We also assume that 
the stochastic process $\Lambda(t)$ satisfies $\lambda^{*}(t):=\mathrm{E} \Lambda(t)<\infty$ for any $t \geq 0$ and $\lambda^{*}(t) \rightarrow \infty$ as $t \rightarrow \infty$, and that, for some $p>\gamma_{G}$ and any $\theta>0$,

$$
\mathrm{E} \Lambda^{p}(t) \mathbf{1}_{(\Lambda(t))>(1+\theta) \lambda^{*}(t)}=O\left(\lambda^{*}(t)\right) .
$$

Let $N_{1}^{*}(t)=\sup \left\{\sigma_{n} \geq t, I_{n}=1\right\}, t \geq 0$, denote the number of claims of the first kind that really occur in the interval $[0, t]$. Clearly,

$$
N_{1}^{*}(t)=\sum_{j=1}^{N_{1}(t)} I_{j} \quad \text { and } \quad \mathrm{E} N_{1}^{*}(t)=q \lambda(t), \quad t \geq 0 .
$$

Now, (5.1) can be rewritten as

$$
S(t)=\sum_{j=1}^{N_{1}^{*}(t)} X_{j}+\sum_{j=1}^{N_{2}(t)} Y_{j}
$$

$\mathrm{Ng}$ et al. (2004) proved that if the i.i.d. interarrival times have finite expectation then Assumption 4.1 holds for the counting process $\left\{N_{1}^{*}(t), t \geq 0\right\}$, and if the stochastic process $\{\Lambda(t)\}$ satisfies (5.2) then Assumption 4.1 holds for the counting process $\left\{N_{2}(t), t \geq 0\right\}$ defined as above. Thus, by Theorem 4.1, we obtain the following precise large deviations result. For any $\gamma>0$, as $t \rightarrow \infty$,

$$
\mathrm{P}\left(S(t)-q \lambda_{1}(t) \mu-v \lambda^{*}(t)>x\right) \sim q \lambda_{1}(t) \bar{F}(x)+\lambda^{*}(t) \bar{G}(x)
$$

uniformly for $x \geq \max \left\{\gamma \lambda_{1}(t), \gamma \lambda^{*}(t)\right\}$.

\section{Acknowledgement}

The authors would like to express their gratitude to an anonymous referee for constructive comments on previous versions of this paper. This work was supported by the National Natural Science Foundation of China (grant number 10771070).

\section{References}

Berman, S. M. (1982). Sojourns and extremes of a diffusion process on a fixed interval. Adv. Appl. Prob. 14, 811-832. Bingham, N. H., Goldie, C. M. and Teugels, J. L. (1987). Regular Variation. Cambridge University Press.

CAI, J. AND TANG, Q. H. (2004). On max-sum equivalence and convolution closure of heavy-tailed distributions and their applications. J. Appl. Prob. 41, 117-130.

Cline, D. B. H. (1994). intermediate regular and $\Pi$ variation. Proc. Lond. Math. Soc. 68, 594-616.

Cline, D. B. H. And Hsing, T. (1991). Large deviation probabilities for sums and maxima of random variables with heavy or subexponential tails. Preprint, Texas A\&M University.

Denuit, M., Lefèvre, C. AND Utev, S. (2002). Measuring the impact of dependence between claims occurrences. Insurance Math. Econom. 30, 1-19.

Heyde, C. C. (1967). A contribution to the theory of large deviations for sums of independent random variables. $Z$. Wahrscheinlichkeitsth. 7, 303-308.

KlÜPPElberg, C. AND MiKosch, T. (1997). Large deviations of heavy-tailed random sums with applications in insurance and finance. J. Appl. Prob. 34, 293-308.

Nagaev, A. V. (1969). Integral limit theorems for large deviations when Cramer's condition is not fulfilled. I. Theory Prob. Appl. 14, 51-64.

Nagaev, S. V. (1973). Large deviations for sums of independent random variables. In Trans. Sixth Prague Conf. Inf. Theory Statist. Decision Functions Random Process, Academia, Prague. pp. 657-674.

Nagaev, S. V. (1979). Large deviations of sums of independent random variables. Ann. Prob. 7, 745-789. 
NG, K. W., TANG, Q. H., YAn, J. A. AND YANG, H. L. (2003). Precise large deviations for the prospective loss process. J. Appl. Prob. 40, 391-400.

Ng., K. W., TANG, Q. H., YAn, J. A. And YAng, H. L. (2004). Precise large deviations for sums of random variables with consistently varying tails. J. Appl. Prob. 41, 93-107.

TANG, Q. H. AND YAN, J. A. (2002). A sharp inequality for the tail probabilities of sums of i.i.d. r.v.'s with dominatedly varying tails. Sci. China Ser. A. 45, 1006-1011.

TANG, Q. H., Su, C., JiAng, T. AND ZHANG, J. S. (2001). Large deviations for heavy-tailed random sums in compound renewal model. Statist. Prob. Lett. 52, 91-100. 